\title{
Die Zukunft kommt auf jeden Fall
}

\section{Werner Bauer}

Dr. med., Präsident des Schweizerischen Instituts für ärztliche Weiter- und Fortbildung SIWF

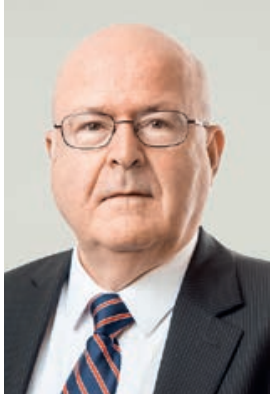

«Die Zukunft des Arztberufes im Wandel der Megatrends» - so lautete der Titel des ersten Hauptreferates am diesjährigen MedEd-Symposium, über welches Bruno Kesseli in dieser Ausgabe der SÄZ (S. 1646-1649) berichtet. Der Referent, Daniel Dettling, Gründer und Leiter des Berliner Büros des Instituts für Zukunftspolitik, stimmte nicht in den oft gehörten Chor vieler Prognostiker ein, die ihre Zuhörer mit Risiken, ungelösten Problemen, Wetterleuchten am Horizont und bedenklichen Entwicklungen eindecken. Nein, er gab zu bedenken, dass viele Menschen eine Optik haben, welche die Welt tendenziell schlechter und die Zukunft düsterer macht, als sie sind, weil die Wachsamkeit gegenüber Gefahren evolutionsgeschichtlich Überlebensvorteile brachte.

Viele Menschen haben eine Optik, welche die Welt tendenziell schlechter und die Zukunft düsterer macht, als sie ist.

Wenn wir also über die Zukunft des Gesundheitswesens und vor allem des Arztberufes nachdenken, müssen wir - so empfahl es der Referent mit Nachdruck - durchaus nüchterne, kluge Einschätzungen vornehmen, ohne uns aber in eine Negativspirale hineinreissen zu lassen. Vieles hat sich geändert, und noch mehr wird sich wohl in Zukunft ändern: Es braucht ein aktualisiertes Berufsbild der Ärztinnen und Ärzte. Die Digitalisierung vieler Prozesse und vor allem die künstliche Intelligenz gehen mit offenen Fragen einher, aber als Reaktion den Modus "Abwehr einer Bedrohung" hochzufahren, sei nicht die Lösung und wäre kontraproduktiv. Die künstliche Intelligenz müsse ihren Sinn nicht im Überflüssigmachen, sondern in der Erweiterung der menschlichen Intelligenz finden. Vieles spreche dafür, dass dies auch geschehen wird.

Trotz aller Verschiebungen von Aktivitäten, trotz des zunehmenden Einsatzes elektronischer Hilfsmittel, von Robotik- und Simulationstechnologie, trotz der Entwicklung des Smartphones zum virtuellen Leibarzt und trotz des ganz anderen Ablaufs von Konsultationen werde der Arztberuf in Zukunft nicht abgewertet. Wohin ihn der Fortschrittsweg führen werde, sei konkret noch nicht absehbar, aber Ärztinnen und Ärzte würden zentrale Funktionen bewahren oder neu übernehmen: als Kommunikatoren und Interpretatoren, als Entscheidungsträger und Koordinatoren (trotz aller Algorithmen), als Vermittler von Gesundheitskompetenz und als diejenigen, welche einen wesentlichen Beitrag für die Weiterentwicklung der Medizin leisten werden.

Der Blick in die Zukunft beschränkte sich am MedEdSymposium, das ja den Perspektiven der ärztlichen Bildung gewidmet ist, nicht auf das Referat von Daniel Dettling. So wurde auch dargestellt, dass die GenderAspekte in der Aus- und Weiterbildung vermehrt berücksichtigt werden müssen. Neue Ausbildungsmethoden, wie die "Entrustable Professional Activities», wurden im Hinblick auf ihre Eignung zur bestmöglichen Vermittlung ärztlicher Kompetenzen hin analysiert. Wie lässt sich die Qualität der Weiterbildung trotz knapper Ressourcen und des Produktivitätsdrucks an den Institutionen sichern? Ein engagierter Chefarzt teilte seine «Rezepte» mit den Zuhörern. Die lebhafte Podiumsdiskussion widmete sich schliesslich der etwas provokativen Frage, ob denn die Weiterbildung an einem Wendepunkt stehe.

Die Anforderungen der Zukunft - wie meistern wir sie denn nun am besten? Kaffeesatzlesen, Extrapolieren von bestehenden Daten, das Akkumulieren von Bedenken und Vorbehalten, ein Korsett von misstrauischen Reglementierungen, das Schielen nach dem

Drei substantielle Empfehlungen für die Zukunft: Neugier, Offenheit und Gestaltungswillen.

Rückwärtsgang - das alles kann's nicht sein. Realistisch betrachtet gibt es immerhin zwei einigermassen substantielle Empfehlungen: Zunächst Neugier, Offenheit und das Vermeiden von "Awfulizing», also der Neigung, die schlimmen Seiten einer Entwicklung $\mathrm{zu}$ überbewerten. Und dann - inzwischen ist dieses Anliegen für mich zum "ceterum censeo" geworden gilt es für uns Ärzte, die Zukunft nicht passiv geschehen zu lassen, sondern uns in die Entwicklungsprozesse aktiv einzubringen und die Zukunft initiativ und kreativ mitzugestalten. Kommen wird sie auf jeden Fall! 